Pak. j. sci. ind. res. Ser. A: phys. sci. 201356 (3) 165-175

\title{
Distribution of Metals in Urban Street Dusts of Benin City, Nigeria
}

\author{
Chukwujindu Maxwell Azubuike Iwegbue ${ }^{\mathrm{a} *}$, Embbey Kaine Ossai ${ }^{\mathrm{a}}$, Chukwudumebi Loretta \\ Overah $^{\mathrm{a}}$, Kingsley Opuene ${ }^{\mathrm{b}}$ and Godwin Ebichime Nwajei ${ }^{\mathrm{a}}$ \\ ${ }^{\mathrm{a}}$ Department of Chemistry, Delta State University, P.M. B 1 Abraka, Nigeria \\ ${ }^{\mathrm{b}}$ Industrial Laboratory, OB/OB Gas Plant, Nigerian Agip Oil Company, Omoku Rivers State, Nigeria
}

(received January 31, 2012, revised November 17, 2012; accepted December 5, 2012)

\begin{abstract}
Dust samples were collected from 30 sites within Benin city, Nigeria, during the month of December, 2006 - March 2007 and analysed for $\mathrm{Cu}, \mathrm{Cr}, \mathrm{Ni}, \mathrm{Cd}$ and $\mathrm{Pb}$ using atomic absorption spectrophotometry. The samples were divided into 3 categories, including a control. Results showed that the dust samples contained significant levels of the metals studied compared to the control site. The mean values for $\mathrm{Cu}, \mathrm{Cr}, \mathrm{Ni}, \mathrm{Cd}$ and $\mathrm{Pb}$ were $16.83 \mathrm{mg} / \mathrm{kg}, 55.40 \mathrm{mg} / \mathrm{kg}, 5.91 \mathrm{mg} / \mathrm{kg}, 3.17 \mathrm{mg} / \mathrm{kg}$, and $182 \mathrm{mg} / \mathrm{kg}$, respectively, for the high traffic density. The mean concentrations of $\mathrm{Cu}, \mathrm{Cr}, \mathrm{Ni}, \mathrm{Cd}$ and $\mathrm{Pb}$ were $11.98 \mathrm{mg} / \mathrm{kg}, 52.21 \mathrm{mg} / \mathrm{kg}, 6.89 \mathrm{mg} / \mathrm{kg}, 3.92 \mathrm{mg} / \mathrm{kg}$ and $167.34 \mathrm{mg} / \mathrm{kg}$, respectively, for the medium traffic areas, while mean concentrations of $\mathrm{Cu}, \mathrm{Cr}, \mathrm{Ni}, \mathrm{Cd}$ and $\mathrm{Pb}$ in the low traffic areas were $10.46 \mathrm{mg} / \mathrm{kg}, 58.7 \mathrm{mg} / \mathrm{kg}, 8.06 \mathrm{mg} / \mathrm{kg}, 3.49 \mathrm{mg} / \mathrm{kg}$ and $142.53 \mathrm{mg} / \mathrm{kg}$, respectively. These values suggest that motor vehicles and electricity generating sets formed the major sources of these metals in the dust samples. The values of metals in the dust samples in these areas were compared with the results of investigations in other countries and these values at various zones of Benin city were found similar, which indicates that Benin city can be considered as one big urban centre with high population and traffic density.
\end{abstract}

Keywords: heavy metals, contamination, dust, vehicular emission; electricity generating sets

\section{Introduction}

Air pollution is a major environmental problem associated with rapid urbanization and industrial growth. Human activities are responsible for the mobilization of metals into the biosphere thereby contributing an important process in the geochemical recycling of these contaminants (Addo et al., 2012). Dust is a complex mixture of particulates with a varied chemical composition resulting from the interact of solid, liquid and gaseous materials produced from different sources, processes and activities (Addo et al., 2012; Hjotenkrans et al., 2006; Singh et al., 2005). Urban dusts have been implicated for its potential to carry high load of myriads of environmental pollutants such as metals, PAHs and PCBs etc. (Faiz et al., 2009; Lu et al., 2009). The occurrence of high concentrations of metals in dust particles are potential hazard to human health especially to children (Yap et al., 2012; Meza-Figueroa et al., 2007) who often play in the street, school yard, parks, etc. The main source of road dust is deposition of atmospheric aerosol particles. In urban environment, these particles originate mainly from road traffic,

*Author for correspondence; E-mail: maxipriestley@yahoo.com emissions from industries, construction activities and from the flaking of paints (Radojevic and Bashkin, 1999). Particles larger than $10 \mu \mathrm{m}$ in diameter are deposited quite rapidly to the earth surface under the influence of gravity. The study of metal in dust particles is important because dust can be freely inhaled by those transversing the streets and those residing within the streets. Dust is one of the major mediums through which metals find its way into soil, surface and groundwater after the rain shower (Tamrakar and Shakya, 2011). In many developing countries, where urban pollution levels are especially high, a large number of children live in the roadsides and spend most of the day on the street under conditions of poor hygiene and subsequently ingests this dust.

Occurrence of metals in the environment is of great concern because some of these metals are essential for normal growth, while others can not be tolerated at even low concentrations due to their toxicity. For example, lead toxicity is characterised by symptoms such as anaemia, colic, neuropathy, sterility and coma. Exposure to low doses of lead has been implicated for behavioural abnormalities, learning disabilities, impaired hearing and cognitive function in human (Chauhan et 
al., 2010). Lead can freely cross the placenta during pregnancy causing intrauterine foetal death, premature delivery and low birth weight (Papanikolaou et al., 2005). Blood lead level of approximately $10 \mu \mathrm{g} / \mathrm{dL}$ could lead to increased risk of pregnancy hypertension, spontaneous abortion and reduced offspring neurobehavioural development (Bellinger, 2005). Cadmium is known to impair kidney function, reproductive capacity, cause hypertension, tumour, hepatic dysfunction (Iwegbue, 2011) and skeletal damage (Jarup, 2003). The toxicity of $\mathrm{Cd}$ is associated with its ability to displace physiologically appropriate metals such as $\mathrm{Cu}$ and $\mathrm{Fe}$ in cytoplasmic and membrane proteins (Marias and Blackhurst, 2009). Chromium (VI) is a known carcinogen. At higher concentrations, $\mathrm{Cr}, \mathrm{Cu}$ and $\mathrm{Zn}$ are associated with toxicity symptoms such as nephritis, anuria and extensive lesions in the kidney (Iwegbue, 2011).

Street dust has several advantages for pollution monitoring, since the heavy metals are present at concentrations that make it possible to obtain samples and analysis without disturbing the ecosystem, which gives room for large scale and repeated sampling (Ayodele and Gaya, 1998).Though there are numerous studies of heavy metal contamination of urban dusts in developed countries, little information is available on heavy metals of urban dusts of developing countries (Banerjee, 2003) including Nigeria, where relatively few studies on metals contamination of urban dusts have been documented (Shinggu et al., 2007; Mashi et al., 2005; Ayodele and Gaya, 1998). The objective of the present study is to determine the concentrations of five metals $(\mathrm{Cu}, \mathrm{Cr}, \mathrm{Ni}, \mathrm{Cd}$ and $\mathrm{Pb})$ in urban street dusts from Benin city with a view of providing information on the sources and risk associated with metal contamination of urban dusts.

\section{Materials and Methods}

Description of study area. The study area (Benin city) lies within the latitude $6^{\circ} .20^{\prime} \mathrm{N}$ and $6^{\circ} .31 ' \mathrm{~N}$ and longitude $5^{\circ} .35^{\prime} \mathrm{E}$ and $5^{\circ} .41^{\prime} \mathrm{E}$. The mean annual rainfall in this area ranges between $2540-3500 \mathrm{~mm}$. In certain years, the mean annual rainfall could rise to above $4000 \mathrm{~mm}$. The temperature for this area ranges between $28^{\circ} \mathrm{C}$ and $33^{\circ} \mathrm{C}$ around March and $23^{\circ} \mathrm{C}$ and $31^{\circ} \mathrm{C}$ in August. The relative humidity of the area is highest in August and ranges between $80-90 \%$. It is lowest between November and March (60-85\%). Two rivers (Ogba and Ikpoba) formed the drainage system of Benin city.
The principal industries in Benin city are brewery, pharmaceutical, food processing company and furniture industries. Other mediums scale industrial activities include welding and fabrication, bronze casting etc.

Sampling. Dust samples were collected from 30 sites within Benin city for a monthly period of December 2006 to March 2007. The high traffic zones had traffic density of 10,000 - 12,000 vehicles/day; medium traffic zones have traffic density of $6000-8,000$ vehicles/day and the low traffic density zones have traffic density less than 5,000 vehicle/day. Samples were collected using a clean plastic dustpan and a brush (Akhter and Madany, 1993). The dust samples were collected at monthly interval for the above period from each site. A control site sample was collected from a rural settlement at the outskirt of the city in Egor Local Government area (the control site has traffic density of less than 50 cars/day). The dust samples were oven dried at $40-60{ }^{\circ} \mathrm{C}$, sieved, and stored in a polyethylene containers at $4{ }^{\circ} \mathrm{C}$ prior to analysis.

Reagents. All reagents used were of analytical grade, working standards of cadmium, chromium, copper, nickel and lead were prepared by diluting concentrated stock solution (Merck Darmstadt, Germany) of 1000 $\mathrm{mg} / \mathrm{L}$ with $0.25 \mathrm{M} \mathrm{HNO}_{3}$.

Sample Preparation. One gram of the dust samples of each site were placed in a Kjeldahl flask and $15 \mathrm{~mL}$ of aqua regia was added and swirled to wet the samples. The samples were allowed to stand overnight. In the next day, the flask was placed in heating block and heated to $50^{\circ} \mathrm{C}$ for $30 \mathrm{~min}$ and raised to $120^{\circ} \mathrm{C}$ and continued for $2 \mathrm{~h}$. The digest was cooled to room temperature and dissolved in $10 \mathrm{~mL}$ of $0.25 \mathrm{M} \mathrm{HNO}_{3}$ and filtered through Whatman No. 1 filter paper and made up to $50 \mathrm{~mL}$ with $0.25 \mathrm{~mol} / \mathrm{L} \mathrm{HNO}_{3}$.

Chemical analysis. All digested samples were analysed in triplicate using graphite flame atomic absorption spectrophotometry (GBC scientific equipment SENS AA, Melbourne, Australia) equipped with $\mathrm{D}_{2}$ background corrective device.

Quality control and statistical analysis of results. Quality control was assured by the use of procedural blanks prepared in a similar manner like the samples and spike recovery method (SRM). Reagent blanks were used to correct all instrument readings. All glassware was cleaned with $10 \% \mathrm{HNO}_{3}$ for $48 \mathrm{~h}$ then rinsed with deionised water. The spike recoveries for each of the elements screened were greater than $93 \%$. 
Results are expressed as mean \pm standard deviation SD and one way analysis of variance was carried out using a statistical analysis system (SPSS version 12). Differences in the concentrations of the elements within a given zone were tested with ANOVA. Turkey multiplecomparison test was used to compare the differences in the mean values of the elements from different zones and principal component analysis was used to establish relationship between the metals.

Contamination assessment methods of the street dust. There are several ways of expressing the contamination levels of soil and sediments. The most common ways include index of geoaccumulation and enrichment factor (Addo et al., 2012). In this study, geoaccumulation (Igeo), enrichment factor (EF), contamination factor (CF) and pollution load index (PLI) have been applied to assess the metal contamination levels of urban street dust of Benin city.

Index of geoaccumulation (Igeo). The index of geoaccumulation (Igeo) was originally used to assess the extent of metal contamination of sediment (Muller, 1969) which has been applied here.

The index of geoaccumulation is given by the formula

$$
\mathrm{I}_{\text {geo }}=\log _{2}\left[\frac{\mathrm{C}_{\mathrm{n}}}{1.5 \mathrm{~B}_{\mathrm{n}}}\right]
$$

where:

$\mathrm{Cn}=$ measured concentration of the metal in the tested sample (street dust)

$\mathrm{B}_{\mathrm{n}}=$ the geochemical background value of the element in fossil argillaceous sediment (continental crusted average or average shale).

However, the mean value of metals at the control site was used as background concentration in this study.

The constant 1.5 is introduced to minimize the effect of possible variations in background values which may be attributed to lithologic variation in the sediment (Addo et al., 2012; Lu et al., 2009).

The interpretation for the geoaccumulation index is as follows: Igeo $<0=$ practically unpolluted; 0 $<$ Igeo $<1=$ unpolluted to moderately polluted; 1 $<$ Igeo $<2=$ moderately polluted, $2<$ Igeo $<3=$ moderately to strongly polluted; $3<$ Igeo $<4=$ strongly polluted; $4<$ Igeo $<5=$ strongly polluted and Igeo $>5=$ extremely polluted to very polluted.

Enrichment factor. Contamination factor (Enrichment factor) and pollution load index. The contamination factor reflects metal enrichment in the dust.

$$
\mathrm{CF}=\mathrm{C}_{\text {metal }} / \mathrm{C}_{\text {background }}
$$

where:

$\mathrm{CF}$ is the contamination factor, $\mathrm{C}_{\text {metal }}$ is the concentration of pollutant in the dust; $\mathrm{C}_{\text {background }}$ is the value of the metals.

In this study, the concentration of metals at the control site was used as the background concentration for the metals, where the contamination factor $\mathrm{CF}<1$ refers to low contamination, $1 \leq \mathrm{CF} \leq 3$ indicate moderate contamination; $3 \leq \mathrm{CF} \leq 6$ indicates considerable contamination and $\mathrm{CF}>6$ indicates very high contamination. Enrichment factor can give an insight to differentiating an anthropogenic source from a natural origin as well as assessment of the degree of metal contamination. Five contamination categories are recognised on the basis of enrichment factor (Loska et al., 2003; Sutherland et al., 2000). The contamination categories based on $\mathrm{EF}$ values include: $\mathrm{EF}<2$ deficiency to minimal enrichment; $\mathrm{EF}=2-5$ moderate enrichment; $\mathrm{EF}=5-20$ significant enrichment; $\mathrm{EF}=20-40$ very high enrichment; $\mathrm{EF}>40$ extremely high enrichment.

Each site was evaluated for the extent of metal pollution using the pollution load index (PLI) developed by Thomilson et al. (1980). The PLI is given by the expression

$$
\mathrm{PLI}=\sqrt[n]{\mathrm{CF}_{1} \times \mathrm{CF}_{2} \times \mathrm{CF}_{3} \times \ldots . . \times \mathrm{XF}_{\mathrm{n}}}
$$

where:

$\mathrm{n}$ is the number of metal studied (five in this study)

The PLI provide simple but comparative means for assessing a site quality, where a value of PLI $<1$ denotes perfection, $\mathrm{PLI}=1$ indicates that only baseline levels of the pollutants are present and PLI $>1$ would indicate deterioration of site quality (Thomilson et al., 1980).

\section{Results and Discussion}

The overall mean concentrations of $\mathrm{Cu}, \mathrm{Cr}, \mathrm{Ni}, \mathrm{Cd}$ and $\mathrm{Pb}$ in the dust samples from high, medium and low traffic density zones are presented in Table 1, while Table 2 presents the mean values for each element at each site in the different zones. The values in parenthesis represent concentration range. Analysis of variance on the average of all data of each location over sampling period (December 2006 - March, 2007) showed no significant difference in the concentrations of copper, chromium, nickel, cadmium and lead at $95 \%$ probability level except for sites $\mathrm{HTD}_{5}$ and $\mathrm{HTD}_{9}$. However, there 
Table 1. Overall mean concentration of metals $(\mathrm{mg} / \mathrm{kg})$ in difference traffic density areas

\begin{tabular}{|c|c|c|c|c|c|c|}
\hline \multirow[b]{2}{*}{ Metals } & \multicolumn{2}{|c|}{ HTD } & \multicolumn{2}{|c|}{ MTD } & \multicolumn{2}{|c|}{ LTD } \\
\hline & Range & Mean & Range & Mean & Range & Mean \\
\hline $\mathrm{Cu}$ & $0.05-43.0$ & $16.83 \pm 12.26$ & $3.20-21.10$ & $11.98 \pm 6.51$ & $5.90-15.90$ & $10.46 \pm 4.02$ \\
\hline $\mathrm{Cr}$ & $0.05-123.0$ & $55.40 \pm 34.30$ & $16.00-92.30$ & $52.21 \pm 25.49$ & $6.80-103.80$ & $58.70 \pm 36.91$ \\
\hline $\mathrm{Ni}$ & $0.05-38.20$ & $5.91 \pm 10.67$ & $0.05-44.20$ & $6.89 \pm 15.42$ & $0.05-22.10$ & $8.06 \pm 9.99$ \\
\hline $\mathrm{Cd}$ & $0.05-11.50$ & $3.17 \pm 3.75$ & $0.05-9.20$ & $3.94 \pm 3.85$ & $0.05-7.10$ & $3.49 \pm 2.89$ \\
\hline $\mathrm{Pb}$ & $83.70-294.30$ & $182.06 \pm 51.44$ & $131.70-195.90$ & $167.34 \pm 23.14$ & $104.4-179.30$ & $142.43 \pm 29.28$ \\
\hline
\end{tabular}

$\mathrm{HTD}=$ high traffic density; MTD = medium traffic density; LTD = low traffic density.

are significant variations in the metal concentrations when various sites were compared. All sites showed higher levels of the metals than the control site except for $\mathrm{Cd}, \mathrm{Cr}$, and $\mathrm{Cu}$ in some sites, which indicates that levels of metals at such sites are not resulting from pollution, rather due to natural background levels.

The mean levels of $\mathrm{Cu}$ in the dust samples ranged from $0.05-43.0 \mathrm{mg} / \mathrm{kg}, 3.20-21.10 \mathrm{mg} / \mathrm{kg}$ and $5.90-15.9$ $\mathrm{mg} / \mathrm{kg}$ for high medium and low traffic density zones, respectively. Significant higher levels of $\mathrm{Cu}$ were observed in sites $\mathrm{HTD}_{7}$ and $\mathrm{HTD}_{3}$ as compared with any other sites. The high traffic density zone recorded higher levels of $\mathrm{Cu}$ as compared with medium and low traffic density zones. Copper in the dust particles are derived from engine wear, from thrust bearing, bushing and bearing metals (Jaradat and Momani, 1999). Other sources of $\mathrm{Cu}$ in the studied areas include contamination from furniture manufacturing industries which are situated along the major runways in the city. It has been noted that chemicals such as copper sulphate, boliden salt (BIS salt) mixed with sulphate and chromate copper arsenate (CCA) have been used as preservatives in wood industries (Bhattacharya et al., 2002). The levels of $\mathrm{Cu}$ found in this study are consistent with the levels reported for urban dusts (El-Hassan et al., 2006; Dandar and Palar, 2003), however, comparatively lower than some other cities in the world (Table 3).

The mean concentrations of $\mathrm{Cr}$ in the dust samples follow the order; low traffic density zone $>$ high traffic density zone $>$ medium traffic density zone. The concentrations of $\mathrm{Cr}$ range between $0.05-123 \mathrm{mg} / \mathrm{kg}$, $16-92.30 \mathrm{mg} / \mathrm{kg}$ and $6.80-108.8 \mathrm{mg} / \mathrm{kg}$ for high, medium and low traffic density zones, respectively. The highest mean levels of $\mathrm{Cr}$ were observed at sites $\mathrm{HTD}_{14}$ and $\mathrm{MTD}_{3}$ and $\mathrm{LTD}_{7}$ for high, medium and low traffic density zones, respectively. Overall, the mean values of $\mathrm{Cr}$ in the three zones are similar. Studies have shown that stainless steel and alloyed steel contains $\mathrm{Fe}, \mathrm{Cr}$, $\mathrm{Co}, \mathrm{Al}$ and/or $\mathrm{Cu}$ and that exhaust emission from both gasoline and diesel vehicles contain variable quantities of the elements (Chong, 1986). However, metal fabrication, woodwork, bronze casting and paint flaking contribute variable amounts of chromium in this city, Table 3 shows the levels of $\mathrm{Cd}, \mathrm{Cu}, \mathrm{Cr}, \mathrm{Pb}$ and $\mathrm{Ni}$ in this study compared with some other cities.

The concentrations of $\mathrm{Ni}$ on the dust samples ranged between $0.05-38.20 \mathrm{mg} / \mathrm{kg}, 0.05-44.0 \mathrm{mg} / \mathrm{kg}$ and $0.05-22.10 \mathrm{mg} / \mathrm{kg}$ for high, medium and low traffic density zones, respectively. Elevated level of $\mathrm{Ni}$ was observed at sites $\mathrm{HTD}_{1}$ and $\mathrm{HTD}_{4}$ for high traffic density zone, while sites $\mathrm{LTD}_{1}$ and $\mathrm{LTD}_{2}$ have higher levels of $\mathrm{Ni}$ compared to any other sites in the low traffic density zone. However, $\mathrm{Ni}$ is a fuel additive as $\mathrm{Pb}$, especially in burning fuel (diesel) that are used for electricity generator in the residential areas (Sheppard et al., 2000). The mean concentrations of $\mathrm{Cd}$ in the dust samples ranged between $0.05-11.50 \mathrm{mg} / \mathrm{kg}, 0.05-9.20 \mathrm{mg} / \mathrm{kg}$, and $0.05-7.10 \mathrm{mg} / \mathrm{kg}$ for high, medium and low traffic zones, respectively. In all, $33 \%$ of the studied sites had $\mathrm{Cd}$ concentrations similar to that of the control sites. The highest level of $\mathrm{Cd}$ was observed at site $\mathrm{HTD}_{11}$ $(11.50 \mathrm{mg} / \mathrm{kg})$. Metal plating, burning of plastics, pigment, burning of fossil fuel, metallurgical process and tyre rubber are major sources of $\mathrm{Cd}$. Cadmium is also found in lubricating oil as part of many additives. It has been reported that the levels of $\mathrm{Cd}$ in car tyres range from $20-90 \mathrm{mg} / \mathrm{kg}$ as associated with $\mathrm{Cd}$ in the process of vulcanisation (Massadeh and Snook, 2000; Jaradat and Momani, 1999). In the absence of major industries in the sampling sites, the levels of $\mathrm{Cd}$ could be due to lubricating oils and/or older tyres, that are frequently used and the rough surfaces of the roads which increase the wearing of tyres. Natural background levels of soil Cd ranged from $0.01-0.7 \mathrm{mg} / \mathrm{kg}$, urban 
Table 2. Mean concentration $(\mathrm{mg} / \mathrm{kg}) \pm \mathrm{SD}$ ranges of heavy metal in dust

\begin{tabular}{|c|c|c|c|c|c|c|}
\hline Zones & Samples site ID & $\mathrm{Cu}$ & $\mathrm{Cr}$ & $\mathrm{Ni}$ & $\mathrm{Cd}$ & $\mathrm{Pb}$ \\
\hline \multirow[t]{17}{*}{ High traffic } & $\mathrm{HTD}_{1}$ & $\begin{array}{l}6.60 \pm 0.98 \\
(5.61-7.95)\end{array}$ & $\begin{array}{l}49.66 \pm 6.45 \\
(13.21-56.11)\end{array}$ & $\begin{array}{l}44.20 \pm 5.30 \\
(38.50-19.50)\end{array}$ & $\begin{array}{l}0.05 \pm 0.01 \\
(0.04-0.06)\end{array}$ & $\begin{array}{l}131.70 \pm 18.44 \\
(113.28-150.14)\end{array}$ \\
\hline & $\mathrm{HTD}_{2}$ & $\begin{array}{l}9.61 \pm 1.63 \\
(7.98-1.24)\end{array}$ & $\begin{array}{l}62.70 \pm 9.78 \\
(52.92-72.48)\end{array}$ & $\begin{array}{l}16.80 \pm 2.18 \\
(14.62-18.98)\end{array}$ & $\begin{array}{l}0.05 \pm 0.02 \\
(0.04-0.07)\end{array}$ & $\begin{array}{l}83.70 \pm 13.39 \\
(70.38-97.09)\end{array}$ \\
\hline & $\mathrm{HTD}_{3}$ & $\begin{array}{l}0.05 \pm 0.01 \\
(0.04-0.06)\end{array}$ & $\begin{array}{l}63.50 \pm 11.43 \\
(52.07-74.95)\end{array}$ & $\begin{array}{l}0.05 \pm 0.01 \\
(0.04-0.06)\end{array}$ & $\begin{array}{l}0.05 \pm 0.01 \\
(0.03-0.06)\end{array}$ & $\begin{array}{l}144.30 \pm 17.32 \\
(126.98-161.62)\end{array}$ \\
\hline & $\mathrm{HTD}_{4}$ & $\begin{array}{l}39.80 \pm 1.78 \\
(35.12-11.58)\end{array}$ & $\begin{array}{l}0.05 \pm 0.01 \\
(0.04-0.06)\end{array}$ & $\begin{array}{l}38.20 \pm 5.73 \\
(32.47-13.93)\end{array}$ & $\begin{array}{l}0.70 \pm 0.13 \\
(0.57-0.83)\end{array}$ & $\begin{array}{l}148.90+19.36 \\
(129.54-168.26)\end{array}$ \\
\hline & $\mathrm{HTD}_{5}$ & $\begin{array}{l}9.60 \pm 1.15 \\
(8.15-1.72)\end{array}$ & $\begin{array}{l}17.80 \pm 15.13 \\
(102.49-133.11)\end{array}$ & $\begin{array}{l}8.80 \pm 1.14 \\
(7.66-9.94)\end{array}$ & $\begin{array}{l}4.50 \pm 0.77 \\
(3.73-5.27)\end{array}$ & $\begin{array}{l}141.60 \pm 21.24 \\
(120.36-162.84)\end{array}$ \\
\hline & $\mathrm{HTD}_{6}$ & $\begin{array}{l}16.66 \pm 1.15 \\
(12.45-20.75)\end{array}$ & $\begin{array}{l}63.70 \pm 8.28 \\
(55.42-71.98)\end{array}$ & $\begin{array}{l}13.10 \pm 1.73 \\
(11.37-14.83)\end{array}$ & $\begin{array}{l}8.20 \pm 1.07 \\
(7.13-9.27)\end{array}$ & $\begin{array}{l}203.70 \pm 36.67 \\
(167.03-240.37)\end{array}$ \\
\hline & $\mathrm{HTD}_{7}$ & $\begin{array}{l}21.70 \pm 3.47 \\
(18.23-25.17)\end{array}$ & $\begin{array}{l}84.10 \pm 10.93 \\
(73.17-95.03)\end{array}$ & $\begin{array}{l}0.05 \pm 0.01 \\
(0.04-0.06)\end{array}$ & $\begin{array}{l}0.05 \pm 0.01 \\
(0.04-0.06)\end{array}$ & $\begin{array}{l}209.60 \pm 3.68 \\
(174.22-245.56)\end{array}$ \\
\hline & $\mathrm{HTD}_{8}$ & $\begin{array}{l}43.00 \pm 5.16 \\
(37.84-48.16)\end{array}$ & $\begin{array}{l}48.50 \pm 4.85 \\
(43.65-53.35)\end{array}$ & $\begin{array}{l}0.05 \pm 0.01 \\
(0.03-0.07)\end{array}$ & $\begin{array}{l}0.05 \pm 0.01 \\
(0.04-0.06)\end{array}$ & $\begin{array}{l}209.90 \pm 33.58 \\
176.32-243.48)\end{array}$ \\
\hline & $\mathrm{HTD}_{9}$ & $\begin{array}{l}27.80 \pm 5.28 \\
(22.53-33.08)\end{array}$ & $\begin{array}{l}66.30 \pm 8.62 \\
(57.68-74.92)\end{array}$ & $\begin{array}{l}0.05 \pm 0.01 \\
(0.04-0.08)\end{array}$ & $\begin{array}{l}0.05 \pm 0.01 \\
(0.03-0.07)\end{array}$ & $\begin{array}{l}209.90 \pm 27.29 \\
(182.6-237.19)\end{array}$ \\
\hline & $\mathrm{HTD}_{10}$ & $\begin{array}{l}9.00 \pm 2.70 \\
(6.30-11.70)\end{array}$ & $\begin{array}{l}34.60 \pm 6.23 \\
(28.37-40.83)\end{array}$ & $\begin{array}{l}0.05 \pm 0.01 \\
(0.03-0.06)\end{array}$ & $\begin{array}{l}3.50 \pm 0.53 \\
(2.97-4.03)\end{array}$ & $\begin{array}{l}184.60 \pm 33.23 \\
(151.37-217.83)\end{array}$ \\
\hline & $\mathrm{HTD}_{11}$ & $\begin{array}{l}6.00 \pm 0.36 \\
(5.64-0.36)\end{array}$ & $\begin{array}{l}0.05 \pm 0.02 \\
(0.01-0.07)\end{array}$ & $\begin{array}{l}0.05 \pm 0.02 \\
(0.04-0.07)\end{array}$ & $\begin{array}{l}11.50 \pm 1.95 \\
(9.90-13.46)\end{array}$ & $\begin{array}{l}294.30 \pm 50.03 \\
(244.27-344.33)\end{array}$ \\
\hline & $\mathrm{HTD}_{12}$ & $\begin{array}{l}14.60 \pm 1.75 \\
(12.85-16.35)\end{array}$ & $\begin{array}{l}64.60 \pm 8.40 \\
(73.00-56.20)\end{array}$ & $\begin{array}{l}9.50 \pm 10.34 \\
(8.15-10.83\end{array}$ & $\begin{array}{l}0.05 \pm 0.01 \\
(0.03-0.06)\end{array}$ & $\begin{array}{l}184.50 \pm 33.20 \\
(151.30-217.70)\end{array}$ \\
\hline & $\mathrm{HTD}_{13}$ & $\begin{array}{l}17.80 \pm 1.78 \\
(16.02-19.58)\end{array}$ & $\begin{array}{l}26.70 \pm 4.27 \\
(22.43-30.97)\end{array}$ & $\begin{array}{l}0.05 \pm 10.02 \\
(0.03-0.06)\end{array}$ & $\begin{array}{l}3.10 \pm 0.56 \\
(2.54-3.66)\end{array}$ & $\begin{array}{l}236.70 \pm 37.87 \\
(198.83-274.57)\end{array}$ \\
\hline & $\mathrm{HTD}_{14}$ & $\begin{array}{l}6.70 \pm 1.05 \\
(5.65-7.75)\end{array}$ & $\begin{array}{l}123.00 \pm 22.14 \\
(100.86-145.14)\end{array}$ & $\begin{array}{l}0.05 \pm 0.02 \\
(0.04-0.07)\end{array}$ & $\begin{array}{l}8.70 \pm 1.22 \\
(7.48-9.92)\end{array}$ & $\begin{array}{l}212.50 \pm 34.00 \\
(178.50-246.50)\end{array}$ \\
\hline & $\mathrm{HTD}_{15}$ & $\begin{array}{l}11.60 \pm 1.97 \\
(9.63-13.57)\end{array}$ & $\begin{array}{l}40.20 \pm 5.63 \\
(4.57-45.83)\end{array}$ & $\begin{array}{l}0.05 \pm 0.01 \\
(0.04-0.06)\end{array}$ & $\begin{array}{l}1.90 \pm 0.27 \\
(1.63-2.24)\end{array}$ & $\begin{array}{l}170.50 \pm 30.69 \\
(139.81-201.19)\end{array}$ \\
\hline & $\mathrm{HTD}_{16}$ & $\begin{array}{l}21.60 \pm 3.24 \\
(18.36-21.84)\end{array}$ & $\begin{array}{l}58.00 \pm 8.70 \\
(19.30-66.70)\end{array}$ & $\begin{array}{l}11.25 \pm 1.46 \\
(9.79-12.71)\end{array}$ & $\begin{array}{l}5.20 \pm 0.88 \\
(4.32-6.08)\end{array}$ & $\begin{array}{l}128.10 \pm 21.78 \\
(106.32-149.88)\end{array}$ \\
\hline & $\mathrm{HTD}_{17}$ & $\begin{array}{l}11.50 \pm 2.07 \\
(9.43-13.57)\end{array}$ & $\begin{array}{l}41.80 \pm 5.43 \\
(36.37-47.23)\end{array}$ & $\begin{array}{l}0.05 \pm 0.02 \\
(0.03-0.07)\end{array}$ & $\begin{array}{l}0.05 \pm 0.01 \\
(0.03-0.06)\end{array}$ & $\begin{array}{l}152.60 \pm 1.3 \mathrm{I} \\
(134.29-170.91)\end{array}$ \\
\hline \multirow{6}{*}{$\begin{array}{l}\text { Medium } \\
\text { traffic } \\
\text { density }\end{array}$} & $\mathrm{MTD}_{1}$ & $\begin{array}{l}9.80 \pm 1.86 \\
(7.94-11.66)\end{array}$ & $\begin{array}{l}29.70 \pm 3.86 \\
(25.81-33.56)\end{array}$ & $\begin{array}{l}0.05 \pm 0.01 \\
(0.05-0.06)\end{array}$ & $\begin{array}{l}9.20 \pm 1.20 \\
(9.00-10.4)\end{array}$ & $\begin{array}{l}160.50 \pm 28.89 \\
(131.61-189.39)\end{array}$ \\
\hline & $\mathrm{MTD}_{2}$ & $\begin{array}{l}18.40 \pm 2.76) \\
(15.65-21.16)\end{array}$ & $\begin{array}{l}65.30 \pm 9.14 \\
(56.16-74.44)\end{array}$ & $\begin{array}{l}0.05 \pm 0.01 \\
(0.04-0.06)\end{array}$ & $\begin{array}{l}9.00 \pm 1.17 \\
(7.83-10.17)\end{array}$ & $\begin{array}{l}179.60 \pm 23.35 \\
(156.25-202.95)\end{array}$ \\
\hline & $\mathrm{MTD}_{3}$ & $\begin{array}{l}21.10 \pm 1.01 \\
(17.09-25.11)\end{array}$ & $\begin{array}{l}92.30 \pm 12.00 \\
(80.30-104.30)\end{array}$ & $\begin{array}{l}1.20 \pm 0.19 \\
(1.01-1.39)\end{array}$ & $\begin{array}{l}5.90 \pm 1.00 \\
(4.90-6.90)\end{array}$ & $\begin{array}{l}189.00 \pm 4.57 \\
(164.43-213.57)\end{array}$ \\
\hline & $\mathrm{MTD}_{4}$ & $\begin{array}{l}5.90 \pm 0.89 \\
(5.01-6.79)\end{array}$ & $\begin{array}{l}16.00 \pm 1.76 \\
(14.24-17.76)\end{array}$ & $\begin{array}{l}0.05 \pm 0.01 \\
(0.03-0.07)\end{array}$ & $\begin{array}{l}3.00 \pm 0.38 \\
(2.62-3.38)\end{array}$ & $\begin{array}{l}195.90 \pm 27.43 \\
(168.47-223.33)\end{array}$ \\
\hline & $\mathrm{MTD}_{5}$ & $\begin{array}{l}3.20 \pm 0.32 \\
(2.88-3.52)\end{array}$ & $\begin{array}{l}69,90 \pm 8.39 \\
(61.51-78.29)\end{array}$ & $\begin{array}{l}0.05 \pm 0.02 \\
(0.01-0.06)\end{array}$ & $\begin{array}{l}4.30 \pm 0.60 \\
(3.70-4.63)\end{array}$ & $\begin{array}{l}151.00 \pm 16.61 \\
(134.39-167.61)\end{array}$ \\
\hline & $\mathrm{MTD}_{6}$ & $\begin{array}{l}16.20 \pm 2.27 \\
(13.93-18.17)\end{array}$ & $\begin{array}{l}30.30 \pm 4.55 \\
(5.75-34.85)\end{array}$ & $\begin{array}{l}0.05 \pm 0.02 \\
(0.03-0.07)\end{array}$ & $\begin{array}{l}0.05 \pm 0.01 \\
(0.04-0.06)\end{array}$ & $\begin{array}{l}146.50 \pm 19.05 \\
(127.45-165.55)\end{array}$ \\
\hline \multirow[t]{7}{*}{$\begin{array}{l}\text { Low traffic } \\
\text { density }\end{array}$} & $\mathrm{LTD}_{1}$ & $\begin{array}{l}13.40 \pm 2.28 \\
(11.1-15.68)\end{array}$ & $\begin{array}{l}62.10 \pm 6.83 \\
(55.27-68.41)\end{array}$ & $\begin{array}{l}20.00 \pm 3.00 \\
(17.00-23.00)\end{array}$ & $\begin{array}{l}7.00 \pm 1.19 \\
(5.81-8.19)\end{array}$ & $\begin{array}{l}139.10 \pm 19.47 \\
(119.63-158.57)\end{array}$ \\
\hline & $\mathrm{LTD}_{2}$ & $\begin{array}{l}5.90 \pm 0.59 \\
(5.31-6.19)\end{array}$ & $\begin{array}{l}70.90 \pm 11.34 \\
(59.56-82.24)\end{array}$ & $\begin{array}{l}22.10 \pm 3.98 \\
(18.12-26.08)\end{array}$ & $\begin{array}{l}0.05 \pm 0.01 \\
(0.04-0.06)\end{array}$ & $\begin{array}{l}162.10 \pm 19.45 \\
(142.65-181.55)\end{array}$ \\
\hline & $\mathrm{LTD}_{3}$ & $\begin{array}{l}14.40 \pm 1.73 \\
(12.67-16.13\end{array}$ & $\begin{array}{l}12.20 \pm 1.34 \\
(10.86-13.54)\end{array}$ & $\begin{array}{l}0.05 \pm 0.01 \\
(0.03-0.06)\end{array}$ & $\begin{array}{l}2.60 \pm 0.31 \\
(2.29-2.92)\end{array}$ & $\begin{array}{l}123.40 \pm 14.81 \\
(108.60-138.21)\end{array}$ \\
\hline & $\mathrm{LTD}_{4}$ & $\begin{array}{l}8.50 \pm 1.36 \\
(7.14-9.86)\end{array}$ & $\begin{array}{l}6.80 \pm 0.88 \\
(5.92-7.68)\end{array}$ & $\begin{array}{l}12.80 \pm 2.05 \\
(10.75-14.85)\end{array}$ & $\begin{array}{l}3.60 \pm 0.47 \\
(3.13-4.07)\end{array}$ & $\begin{array}{l}104.40 \pm 15.66 \\
(88.74-12 J .06)\end{array}$ \\
\hline & $\mathrm{LTD}_{5}$ & $\begin{array}{l}15.90 \pm 2.39 \\
(13.51-19.29)\end{array}$ & $\begin{array}{l}91.90 \pm 13.79 \\
(78.11-105.69)\end{array}$ & $\begin{array}{l}0.05 \pm 0.01 \\
(0.04-0.06)\end{array}$ & $\begin{array}{l}4.00 \pm 0.60 \\
(3.40-4.60)\end{array}$ & $\begin{array}{l}172.70 \pm 24.18 \\
(148.52-196.88)\end{array}$ \\
\hline & $\mathrm{LTD}_{6}$ & $\begin{array}{l}6.70 \pm 0.87 \\
(5.83-7.57)\end{array}$ & $\begin{array}{l}63.20 \pm 7.58 \\
(55.62-70.78)\end{array}$ & $\begin{array}{l}0.05 \pm 0.01 \\
(0.04-0.06\end{array}$ & $\begin{array}{l}0.05 \pm 0.01 \\
(0.04-0.06)\end{array}$ & $\begin{array}{l}116.70 \pm 17.51 \\
(99.19-134.21)\end{array}$ \\
\hline & $\mathrm{LTD}_{7}$ & $\begin{array}{l}8.40 \pm 1.18 \\
(7.22-9.58)\end{array}$ & $\begin{array}{l}108.80 \pm 14.4 \\
(24.39-123.21)\end{array}$ & $\begin{array}{l}1.40 \pm 0.17 \\
(1.23-1.57)\end{array}$ & $\begin{array}{l}7.10 \pm 1.14 \\
(5.96-8.24)\end{array}$ & $\begin{array}{l}179.30 \pm 23.3 ! \\
(155.99-202.61)\end{array}$ \\
\hline Control site & & $\begin{array}{l}4.80 \pm 0.86 \\
(3.94-5.66)\end{array}$ & $\begin{array}{l}3.80 \pm 0.58 \\
3.42-4.18)\end{array}$ & $\begin{array}{l}0.05 \pm 0.01 \\
(0.04-0.06)\end{array}$ & $\begin{array}{l}0.05 \pm 0.01 \\
(0.04-0.06)\end{array}$ & $\begin{array}{l}20.65 \pm 1.03 \\
(19.62-21.68)\end{array}$ \\
\hline
\end{tabular}


Table 3. Distribution of metals $(\mathrm{mg} / \mathrm{kg}$ ) in dust particles in some countries of the world

\begin{tabular}{lllllll}
\hline \hline Country/City & $\mathrm{Pb}$ & $\mathrm{Cd}$ & $\mathrm{Cu}$ & $\mathrm{Ni}$ & $\mathrm{Cr}$ & Reference \\
\hline Benin city (Nigeria) & $83.70-294.30$ & $0.05-11.5$ & $0.05-43.0$ & $0.05-44.20$ & $0.04-123.0$ & This study \\
Turkey (Adapazari) & 25.9 & 0.7 & 14.2 & 40.2 & 10.25 & Dandar \& Palar, 2003 \\
Kuwait (Bahrain) & 697.2 & 72.0 & - & 125 & 144 & Akhter \& Madany,1993 \\
United Kingdom & 266 & 4.54 & - & - & - & Massadeh \& Snook, 2000 \\
Jordan & $25.09-75.33$ & $2.36-2.83$ & 14.33 & $17.36-22$ & $14.1-18$ & El- Hassan et al., 2006 \\
Australia (Jackson) & 487 & - & 164 & 27 & 34 & Birch and Scolen, 2003 \\
Nigeria (Mubi) & $20.2-35.37$ & $0.59-1.33$ & 11.63 & nd-8.62 & $\mathrm{d}-5.40$ & Shinggu et al., 2007 \\
Poland (Kazowiekie) & 576.9 & 10.8 & 521.6 & 40 & 295 & Krolak, 2000 \\
Turkey (Istanbul) & 368 & 0.3 & 191.1 & 27.1 & - & Yetimoglu et al., 2007 \\
Turkey (Kayseri) & $49-381$ & 1.45 & $12-315$ & $23-85$ & $31-39$ & Divrkli et al., 2005. \\
China & 230.52 & - & 94.98 & - & 167.28 & Yomgming et al., 2006 \\
Mexico & 32.113 & 2.112 & - & - & - & Benin et al., 1999 \\
Nigeria (Gwagwalada) & 210 & 3.9 & 97 & - & - & Mashi et al., 2005 \\
China (Boaji) & 433.2 & - & 123.2 & 48.8 & 126.7 & Lu et al., 2010 \\
Ghana (Ketu-South) & 22.89 & - & 60 & 73.45 & 744.5 & Addo et al., 2012 \\
Nepal (Kathmandu) & $12.3-116.8$ & $0.3-39.6$ & - & $4.9-86.3$ & $1.4-14.3$ & Tamrakar and Shakya, 2011 \\
Bangladesh (Dhaka City) & 54 & - & 105 & 35 & 135 & Ahmed and Ishiga, 2006 \\
\hline \hline
\end{tabular}

value range from $<0.01-8.0 \mathrm{mg} / \mathrm{kg}$, superfund cleanup goal are $2-20 \mathrm{mg} / \mathrm{kg}$. The levels of Cd observed in the three zones did not exceed the superfund cleanup goal of $20 \mathrm{mg} / \mathrm{kg}$.

In high traffic density zone, higher mean values of $\mathrm{Pb}$ were observed at sites $\mathrm{HTD}_{11}, \mathrm{HTD}_{13}, \mathrm{HTD}_{14}$ with mean concentrations of $294.3 \mathrm{mg} / \mathrm{kg}, 236.7 \mathrm{mg} / \mathrm{kg}$, $212.5 \mathrm{mg} / \mathrm{kg}$, respectively. In the medium traffic zone, higher mean values of lead were observed at sites $\mathrm{MTD}_{4}$ $195.9 \mathrm{mg} / \mathrm{kg}$ and $\mathrm{MTD}_{3} 189.0 \mathrm{mg} / \mathrm{kg}$, respectively. The high traffic density zone showed overall higher mean level of $\mathrm{Pb}$ in comparison with the medium and low traffic density zones. The major sources of lead in the dust samples are due to vehicular emission. Lead tetraethyl has been used as an anti-knock agent in gasoline in Nigeria. The similarity in the concentrations of lead in the various zones indicated that Benin city can be considered as one big urban centre with high population and traffic density. Due to the epileptic power supply in the city, most households and business outfits uses various kinds of electricity generators that uses gasoline which contribute significant amounts of lead to the urban environment. Yetimoglu et al. (2007) reported mean lead concentration of $368.3 \mathrm{mg} / \mathrm{kg}$ in street dusts collected from Pandik to Levent in E-5 in Istanbul, Turkey.

Index of geoaccumulation, enrichment factor and pollution lood index. Geoaccumulation index, enrichment factor and the pollution load index of metals at the examined sites are presented in Table 4. The Igeo for $\mathrm{Cu}$ at these sites ranged between -7.17 to 2.47 for high traffic density areas. In the high traffic density zones, only six sites had Igeo value less than 1 . The Igeo value of copper in the medium traffic areas ranged from -1.17 to 1.55 and -0.29 to 1.14 for low traffic density areas. In overall, 16 out of 30 sites examined had Igeo values $<1$, while 14 sites had Igeo value $>1$ $<3$, which falls within the moderately strongly polluted range.

Two sites had Igeo values for $\mathrm{Cr}<1$. The Igeo values for $\mathrm{Cr}$ ranged between -6.83 and 4.43. Nineteen sites had Igeo value $>3$. Based on the Igeo classification, majority of these sites were strongly polluted with $\mathrm{Cr}$. The Igeo values for Ni ranged from - $0.59-9.2$ with 16 sites having Igeo values $<1$ and 12 sites had Igeo values for $\mathrm{Ni}>5$ (i.e. in the extremely polluted range), while two sites had Igeo values of $\mathrm{Ni}>4<5$. The Igeo values for $\mathrm{Cd}$ range between -0.59 and 7.26. Majority of these sites (20 sites) had the Igeo values for $\mathrm{Cd}>3$, while all other sites had Igeo values $<1$. In the case of $\mathrm{Pb}$, all sites in the three zones had Igeo value ranging from 1.43 to 2.93 . On the basis of Igeo classification, these sites can be classified as moderately to strongly polluted with $\mathrm{Pb}$.

Enrichment factor (EF) can give an insight to differentiating anthropogenic sources from a natural origin as well as assessing the degree of metal contamination. The concentration factors (CF) values for metals ranged from 1.23-8.96, 0.01-32.37, 1.00-4.42, 1.00-844 and 1.0-174 for $\mathrm{Cu}, \mathrm{Cr}, \mathrm{Ni}, \mathrm{Cd}$ and $\mathrm{Pb}$, respectively. According to Zhang and Liu (2002), the EF values between $0.5-1.5$ indicates that the metal is entirely from crusted material or natural processes, whereas, EF 
Table 4. The index of geoaccumulation (Igeo), concentration factor (CF) and pollution load index (PLI) of metal in urban street dust of Benin city, Nigeria

\begin{tabular}{|c|c|c|c|c|c|c|c|}
\hline Site & & $\mathrm{Cu}$ & $\mathrm{Cr}$ & $\mathrm{Ni}$ & $\mathrm{Cd}$ & $\mathrm{Pb}$ & PLI \\
\hline \multirow[t]{2}{*}{$\mathrm{HTD}_{1}$} & Igeo & -0.13 & 3.12 & 9.2 & -0.59 & 2.09 & \\
\hline & $\mathrm{CF}$ & 1.40 & 13.1 & 844.0 & 1.0 & 6.38 & 10.1 \\
\hline \multirow[t]{2}{*}{$\mathrm{HTD}_{2}$} & Igeo & 0.42 & 3.46 & 7.81 & -0.59 & 1.43 & \\
\hline & $\mathrm{CF}$ & 2.00 & 16.50 & 336.0 & 1.0 & 4.05 & 8.52 \\
\hline \multirow[t]{2}{*}{$\mathrm{HTD}_{3}$} & Igeo & -7.17 & 3.49 & -0.59 & -0.59 & 2.22 & \\
\hline & $\mathrm{CF}$ & 0.01 & 16.71 & 1.0 & 1.0 & 6.99 & 1.03 \\
\hline \multirow[t]{2}{*}{$\mathrm{HTD}_{4}$} & Igeo & 2.47 & -6.83 & 8.99 & 3.22 & 2.27 & \\
\hline & $\mathrm{CF}$ & 8.29 & 0.01 & 764.0 & 14.0 & 7.21 & 6.08 \\
\hline \multirow{2}{*}{$\mathrm{HTD}_{5}$} & Igeo & 0.42 & 1.64 & 6.88 & 5.91 & 2.19 & \\
\hline & $\mathrm{CF}$ & 2.00 & 4.68 & 176.0 & 90.0 & 6.86 & 15.90 \\
\hline \multirow[t]{2}{*}{$\mathrm{HTD}_{6}$} & Igeo & 1.21 & 3.48 & 7.45 & 6.77 & 2.72 & \\
\hline & $\mathrm{CF}$ & 3.47 & 16.76 & 262.0 & 164.0 & 9.86 & 30.1 \\
\hline \multirow[t]{2}{*}{$\mathrm{HTD}_{7}$} & Igeo & 1.59 & 3.88 & -0.59 & 0.59 & 2.8 & \\
\hline & $\mathrm{CF}$ & 4.52 & 22.13 & 1.00 & 1.00 & 10.15 & 3.99 \\
\hline \multirow[t]{2}{*}{$\mathrm{HTD}_{8}$} & Igeo & 2.58 & 3.09 & -0.59 & 0.59 & 2.76 & \\
\hline & $\mathrm{CF}$ & 8.96 & 12.76 & 1.00 & 1.00 & 10.17 & 4.10 \\
\hline \multirow[t]{2}{*}{$\mathrm{HTD}_{9}$} & Igeo & 1.95 & 3.54 & -0.59 & -0.59 & 2.76 & \\
\hline & $\mathrm{CF}$ & 5.79 & 17.45 & 1.00 & 1.00 & 10.17 & 4.00 \\
\hline \multirow{2}{*}{$\mathrm{HTD}_{10}$} & Igeo & 0.33 & 2.60 & -0.59 & 5.55 & 2.58 & \\
\hline & $\mathrm{CF}$ & 1.88 & 9.11 & 1.00 & 70.0 & 8.94 & 6.40 \\
\hline \multirow[t]{2}{*}{$\mathrm{HTD}_{11}$} & Igeo & -0.26 & -6.83 & -0.59 & 7.26 & 3.25 & \\
\hline & $\mathrm{CF}$ & 1.25 & 0.01 & 1.00 & 23.0 & 14.25 & \\
\hline \multirow[t]{2}{*}{$\mathrm{HTD}_{2}$} & Igeo & 1.02 & 3.50 & 6.99 & -0.59 & 2.58 & \\
\hline & $\mathrm{CF}$ & 3.04 & 17.0 & 190.0 & 1.0 & 8.93 & 9.74 \\
\hline \multirow[t]{2}{*}{$\mathrm{HTD}_{3}$} & Igeo & 1.31 & 2.23 & -0.59 & 5.97 & 2.93 & \\
\hline & $\mathrm{CF}$ & 3.1 & 7.03 & 1.0 & 62.0 & 11.46 & 7.14 \\
\hline \multirow[t]{2}{*}{$\mathrm{HTD}_{14}$} & Igeo & -0.10 & 4.43 & -0.59 & 6.86 & 2.78 & \\
\hline & $\mathrm{CF}$ & 1.40 & 32.37 & 1.0 & 174.0 & 10.29 & 9.59 \\
\hline \multirow[t]{2}{*}{$\mathrm{HTD}_{15}$} & Igeo & 0.69 & 2.82 & -0.59 & 4.66 & 2.46 & \\
\hline & $\mathrm{CF}$ & 2.42 & 10.58 & 1.0 & 38.0 & 8.26 & 6.03 \\
\hline $\mathrm{HTD}_{16}$ & Igeo & 1.58 & 3.35 & 7.23 & 6.12 & 2.05 & \\
\hline & $\mathrm{CF}$ & 4.50 & 5.26 & 25.0 & 104.0 & 6.20 & 16.17 \\
\hline $\mathrm{HTD}_{17}$ & Igeo & 0.68 & 2.88 & -0.59 & -0.59 & 2.30 & \\
\hline & $\mathrm{CF}$ & 2.40 & 11.00 & 1.00 & 1.00 & 7.39 & 2.87 \\
\hline $\mathrm{MTD}_{1}$ & Igeo & 0.45 & 2.38 & -0.59 & 6.94 & 2.37 & \\
\hline & $\mathrm{CF}$ & 2.04 & 7.82 & 1.00 & 184.0 & 7.77 & 7.44 \\
\hline $\mathrm{MTD}_{2}$ & Igeo & 1.35 & 3.52 & -0.59 & 6.91 & 2.54 & \\
\hline & $\mathrm{CF}$ & 3.83 & 17.18 & 1.00 & 180.0 & 8.70 & 10.06 \\
\hline $\mathrm{MTD}_{3}$ & Igeo & 1.55 & 4.02 & 4.0 & 6.30 & 2.61 & \\
\hline & $\mathrm{CF}$ & 4.40 & 24.3 & 24.0 & 118.0 & 9.15 & 19.43 \\
\hline $\mathrm{MTD}_{4}$ & Igeo & -0.29 & 1.49 & -0.59 & 5.32 & 2.66 & \\
\hline & $\mathrm{CF}$ & 1.23 & 4.21 & 1.0 & 60.0 & 9.49 & \\
\hline $\mathrm{MTD}_{5}$ & Igeo & -1.17 & 3.62 & -0.59 & 5.84 & 2.29 & \\
\hline & $\mathrm{CF}$ & 0.67 & 18.4 & 1.0 & 86.0 & 7.31 & 6.00 \\
\hline $\mathrm{MTD}_{6}$ & Igeo & 1.17 & 2.41 & -0.59 & -0.59 & 2.24 & \\
\hline & $\mathrm{CF}$ & 3.38 & 7.97 & 1.0 & 1.0 & 7.33 & 2.88 \\
\hline $\mathrm{LTD}_{1}$ & Igeo & 0.99 & 3.45 & 8.06 & 6.44 & 2.17 & \\
\hline & $\mathrm{CF}$ & 2.79 & 16.34 & 4.00 & 140 & 6.74 & 28.0 \\
\hline $\mathrm{LTD}_{2}$ & Igeo & -0.29 & 3.64 & 8.20 & -0.59 & 2.39 & \\
\hline & $\mathrm{CF}$ & 1.23 & 18.66 & 442.0 & 1.0 & 7.85 & 9.5 \\
\hline $\mathrm{LTD}_{3}$ & Igeo & 1.00 & 1.10 & -0.59 & 5.12 & 1.99 & \\
\hline & $\mathrm{CF}$ & 3.00 & 3.21 & 1.0 & 52.0 & 5.98 & 4.96 \\
\hline $\mathrm{LTD}_{4}$ & Igeo & 0.24 & 0.26 & 7.42 & 5.59 & 1.75 & \\
\hline & $\mathrm{CF}$ & 1.77 & 1.79 & 256 & 72.0 & 5.06 & 12.6 \\
\hline $\mathrm{LTD}_{5}$ & Igeo & 1.14 & 4.01 & -0.59 & 5.74 & 2.48 & \\
\hline & $\mathrm{CF}$ & 3.31 & 24.18 & 1.00 & 80.0 & 8.36 & 8.83 \\
\hline $\mathrm{LTD}_{6}$ & Igeo & -0.10 & 3.47 & -0.59 & -0.59 & 1.91 & \\
\hline & $\mathrm{CF}$ & 1.40 & 16.63 & 1.00 & 1.00 & 5.65 & 2.65 \\
\hline $\mathrm{LTD}_{7}$ & Igeo & 0.22 & 4.26 & 4.22 & 6.57 & 2.538 & \\
\hline
\end{tabular}


greater than 1.5 suggests that the source is more likely to be anthropogenic.

The pollution load index (PLI) effectively ranks the pollution status of each site. The PLI values for these sites were greater than 1 , indicating deterioration of site quality. Based on PLI values site $\mathrm{HTD}_{6}$ had the highest pollution load compared to other examined sites.

Principal component analysis. A principal component analysis (PCA) was applied to the data sets and the values are reported in Table 5. PCA provides a means of reducing the complexity of the total metal data sets. Principal component loading values provide information on the relationship among the variables (Meglen, 1992). In this study, PCA was applied to study the correlation among the heavy metals. Three principal components were extracted from dust data set at high traffic density area (Table 5). However, the composition of the factors was different. In dust at high traffic density area, group 1 (or factor 1, expressing about $37.52 \%$ of the total variance) includes metals that are mainly from anthropogenic activities $(\mathrm{Cd}$ and $\mathrm{Pb}$ ), group 2 (explaining about $24.91 \%$ of the total variance) returned $\mathrm{Cu}$ and group 3 (or factor 3, expressing about 20.66 of the total variance) returned $\mathrm{Ni}$ alone, which were from anthropogenic activities as well (Knox et al., 1999). The presence of $\mathrm{Cd}$ and $\mathrm{Pb}$ in the same group for dust at high traffic density area reflects similar behaviour or common source as illustrated in Fig.1. For samples from the low traffic region, PCA results show that two principal components were extracted and accounted for $42.56 \%$ and $27.27 \%$ of the total variance (Table 5). Group 1 or factor 1 had significant positive loadings in $\mathrm{Cr}$ and $\mathrm{Pb}$, respectively. These metals are usually from anthropogenic input (Knox et al., 1999). Group 2 or factor 2 had significant positive loadings in $\mathrm{Cu}$ and $\mathrm{Cd}$, which might originate from organic manure, tyres and brake linings as illustrated in Fig.2. Meanwhile, PCA of the heavy metals from the region of medium traffic resulted in one principal component that was extracted. The extraction of one PC reflects similar behaviour or suggests common source (Knox et al., 1999).

Risk assessment. Risk assessment with regards to metal-contaminated dust ingestion was carried out to estimate non-cancer toxic (chronic) risk of the general public including children living in Benin city. Estimation of risk was calculated on the equations detailed in USEPA's Exposure factor Handbook (Hague et al., 2008; Leung et al., 2008; USEPA, 1989). The target

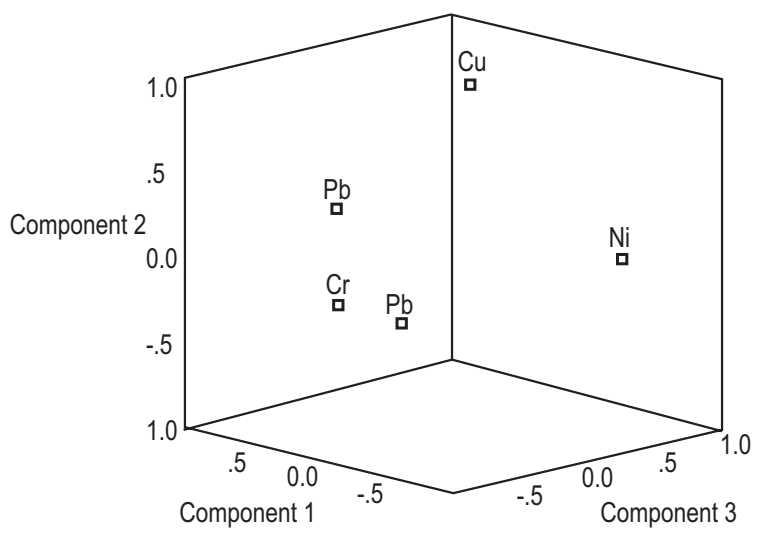

Fig. 1. Component plot in the rotated space for metals in high traffic density area.

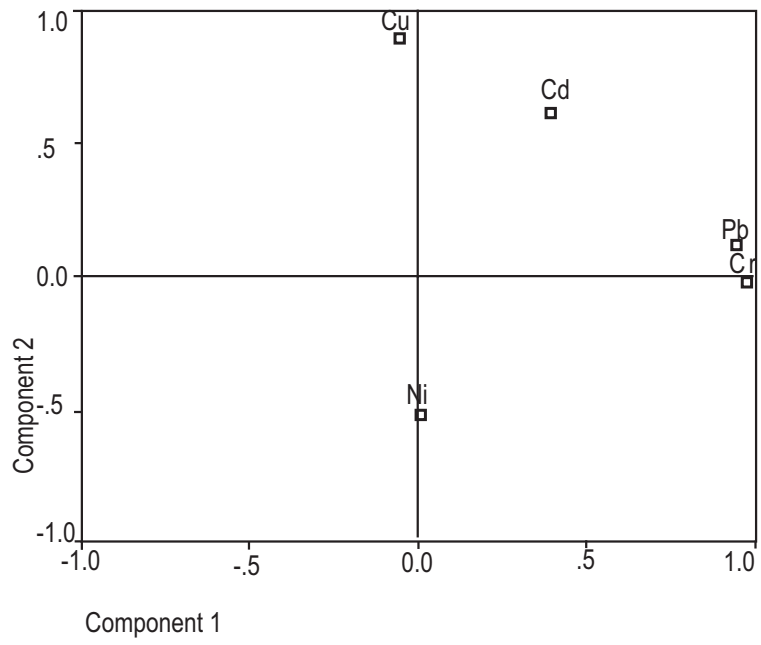

Fig. 2. Component plot in the rotated space for metals in low traffic density area.

hazard quotient (THQ) was determined by the following equation:

$$
\mathrm{THQ}=\frac{\mathrm{C} \times \mathrm{Ing} \mathrm{R} \times \mathrm{EF} \times \mathrm{ED}}{\mathrm{BW} \times \mathrm{AT} \times \mathrm{RFD}} \times 10^{-3}
$$

where:

$\mathrm{C}$ is the mean metal concentration $(\mathrm{mg} / \mathrm{kg})$ in dust. Conservative estimates of dust ingestion rate, Ing R, were chose for adult (100 mg/day) and children (200 $\mathrm{mg}$ /day). An average body weight, BW, of $60 \mathrm{~kg}$ for adults and $15 \mathrm{~kg}$ for children. In this study exposure frequency $\mathrm{EF}=365$ days per year: exposure duration $\mathrm{ED}=6$ years and the averaging time $\mathrm{AT}=2190$ days for children and $\mathrm{ED}=24$ years for adult. RFD is an estimate of daily exposure to human population (including sensitive sub groups) that is likely to be without an appreciable risk of deleterious effect during 
Table 5. PCA factor loadings after varimax rotation with Kaiser normalization for dust at high and low traffic density areas

\begin{tabular}{llllll}
\hline \hline \multirow{2}{*}{ Metals } & \multicolumn{2}{l}{$\begin{array}{l}\text { High traffic density } \\
\text { principal components }\end{array}$} & & \multicolumn{2}{l}{$\begin{array}{l}\text { Low traffic density } \\
\text { principal components }\end{array}$} \\
\cline { 2 - 5 } & Factor 1 & Factor 2 & Factor 3 & Factor 1 & Factor 2 \\
\hline $\mathrm{Cu}$ & - & .927 & .186 & - & .907 \\
$\mathrm{Cr}$ & - & -.110 & -.893 & - & - \\
$\mathrm{Ni}$ & -.694 & - & .529 & .397 & -.526 \\
$\mathrm{Cd}$ & .642 & -.571 & .256 & .949 & .624 \\
$\mathrm{~Pb}$ & .932 & - & - & 42.56 & .106 \\
$\mathrm{Var}(\%)$ & 37.52 & 24.91 & 20.66 & 27.27 \\
\hline \hline
\end{tabular}

Table 6. Estimated target hazard quotient for metals in dust

\begin{tabular}{|c|c|c|c|c|c|c|}
\hline \multirow[t]{2}{*}{ Metals } & \multicolumn{2}{|c|}{$\begin{array}{l}\text { High traffic } \\
\text { density zone }\end{array}$} & \multicolumn{2}{|c|}{$\begin{array}{l}\text { Medium traffic } \\
\text { density zone }\end{array}$} & \multicolumn{2}{|c|}{$\begin{array}{l}\text { Low traffic } \\
\text { density zone }\end{array}$} \\
\hline & Adult & Children & Adult & Children & Adult & Children \\
\hline $\mathrm{Cu}$ & 0.26 & 2.05 & 0.18 & 1.45 & 0.16 & 1.27 \\
\hline $\mathrm{Cr}$ & 0.03 & 0.18 & 0.02 & 0.17 & 0.02 & 0.19 \\
\hline $\mathrm{Cd}$ & 1.93 & 15.43 & 2.40 & 19.2 & 2.12 & 17.0 \\
\hline $\mathrm{Ni}$ & 1.80 & 14.38 & 0.15 & 1.43 & 0.24 & 1.96 \\
\hline $\mathrm{Pb}$ & 0.07 & 0.59 & 0.07 & 0.54 & 0.06 & 0.46 \\
\hline Combined THQ & 4.09 & 32.64 & 2.82 & 22.79 & 2.60 & 20.88 \\
\hline
\end{tabular}

life time. Therefore, THQ $\leq 1$ suggests unlikely adverse health effect where THQ $>1$ suggests the possibility of adverse health effects. A THQ $>10$ is considered to be high chronic risk. It was also assumed that the toxic risks due to metals were additive, therefore the THQ for each metal at the zone scenario was summed to give the combined hazard quotients.

The computed target hazard quotients (THQ) using the mean value for the dust ingest pathway for adult and child scenarios is displayed in Table 6.Of the five metals studied, the THQ for Cd was highest at medium traffic density zone. Generally, the THQ for Cd was highest in all zones (Table 6) for both i.e., adult and child scenarios. In the three zones, the THQ values for $\mathrm{Cd}$ spanned between 15.43 and 19.2. There is a high chronic risk of $\mathrm{Cd}$ for the child scenarios at all zone since the THQ value $>10$. The combined THQ for the metals were greater than 2.60 but less than 4.09 and greater than 20.88 but less than 32.62 for the adult and child scenarios, respectively. The combined THQ for the metals exceeded 1 in all zones which is significant contribution for $\mathrm{Cd}$.

\section{Conclusion}

The metals concentration in dust samples of different zones of Benin city, Nigeria were studied from health point of view and metal contamination were higher in all samples except the control site which illustrates high anthropogenic sources at these zones. The distribution and concentration of heavy metals in all studied areas showed that automobile-originated (emissions or by wear and tear of different parts of the car) and emissions from electricity generators and metal fabrication are the main sources of pollution. The levels of metals found in this study were below the USEPA superfund site cleanup goal. The combined THQ value for these metals indicates a long term risk to human from exposure to these metals particularly $\mathrm{Cd}$.

\section{References}

Addo, M.A., Darko, E.O., Gordon, C., Nyarko, B.J.B., Gbadago, J.K. 2012. Heavy metal concentrations in road deposited dust at Ketu-south district, Ghana. International Journal of Science and Technology, 2: 28-39. 
Ahmed, F., Ishiga, H. 2006. Trace metal concentrations in street dust of Dhaka city, Bangladesh. Atmospheric Environment, 40: 3835-3844.

Akhter, M.S. , Madany, I. M. 1993. Heavy metals in street and household dust in Bahrain. Water, Air, Soil Pollution, 66: 111-119.

Ayodele, J.I., Gaya U.M. 1998. Chromium, zinc and manganese in Kano municipality street dust. Journal of Chemical Society of Nigeria, 23: 24-31.

Barnerjee, A.D.K. 2003. Heavy metal levels and solid phase speciation in street dusts of Delhi, India. Environmental Pollution, 123: 95-105.

Bellinger, D.C. 2005. Teratogen update: levels and pregnancy. Birth Defects Research. Part A: Clinical and Molecular Teratology, 73: 409-420.

Benin, A.L., Sargent, J.D., Dalton, M., Roda, S. 1999. High concentrations of heavy metals in neighbourhoods near ore smelters in northern Mexico. Environmental Health Perspective, 10: 279-284.

Bhattarcharya, P., Mukherjee, A.B., Jack, G., Nordquist, S. 2002. Metal contamination at wood preservation site; characterization and experimental studies on remediation. Science of the Total Environment, 290:165-180.

Birch, G.F., Scolen, A. 2003. Heavy metals in road dust, gully pots and parkland soils in a highly urbanized sub-catchment of Port Jackson, Australia. Australian Journal of Soil Research, 41: 1329-1342.

Chauhan, A.S., Bhadauria, R., Singh, A.K., Lodhi, S.S., Chaturvedi, D.K., Tomar, V.S. 2010. Determination of lead and cadmium in cosmetic products. Journal of Chemical and Pharmaceutical Research, 2: 92-97.

Chong, C. 1986. Determination of silver, bismuth, cadmium, copper, iron, nickel and zinc in lead and tin base solder and white metal bearing alloys by atomic absorption spectrophotometer. Talanta, 33: 91-94.

Divrkli, U., Soylak, M., Elci, L., Dogan, M. 2005. The investigation of trace metal concentration in street dust samples collected from Kayseri, Turkey. Instrumentation Science and Technology, 21: 713-720.

Dunda, M.S., Pala, M.F. 2003. Monitoring of lead, zinc, cadmium, nickel, chromium and copper in street dust samples in Adapazari, Turkey, after earthquake. Trace Elements and Electrolytes, 2: 104-107.

El-Hassan, T., Batarseh, M.A., Oman, H., Ziadat, A., El-Alali, A., Al-Naser, F., Beridanier, B.W., Jiries, A. 2006. The distribution of heavy metals in urban street dusts of Karak city, Jordan. Soil and Sediment Contamination, 15: 357- 365.

Faiz, Y., Tufail, M., Javed, M.T.,Chaudhry, M.M., Siddique, N., 2009. Road dust pollution of $\mathrm{Cd}, \mathrm{Cu}$, $\mathrm{Pb}$ and $\mathrm{Zn}$ along Islamabad expressway, Pakistan. Microchemical Journal, 92: 186-192.

Hague, T., Petroczi, A., Andrews, P.L.R., Barker, J., Naughton, D.P. 2008. Determination of metal in content of beverages and estimation of target hazard quotients: a comparative study. Chemistry Central Journal, 2:13.

Hjortenkrans, D., Bergboak, B., Haggerud, C. 2006. New emission pattern in road traffic environment. Environmental Monitoring and Assessment, 117: 85-98.

Iwegbue, C.M.A. 2011. Concentrations of selected metals in candies and chocolates consumed in southern Nigeria. Food Additives and Contaminants, Part B 4: 22-27.

Jaradat, Q.M., Momani, K.A. 1999. Contamination of roadside soil, plants and air with heavy metals in Jordan, a comparative study. Turkish Journal of Chemistry, 23: 209-220.

Jarup, L. 2003. Hazards of heavy metal contamination. British Medical Bulletin, 68: 167-182.

Knox, A.S., Gamerdinger, A.P., Adriano, D.C. 1999. Source and practices contributing to soil contamination. In: Bioremediation of the Contaminated Soils, D.C. Adriano, J.M. Bollag, and W.T. Frankenberg, (eds.), ASA, CSSA. Agronomy series, No. 37 pp. 53-87, Madison, Wisconsin, USA.

Krolak, E. 2000. Heavy metals in falling dust in Eastern Mazowieckie province. Polish Journal of Environmental Studies, 9: 517-522.

Leung, A.O.W., Duzgoren-Aydis, A.S., Cheung K.C., Wong, M.H., 2008. Heavy metals concentration of surface dust from e-waste recycling and human health implications in southwest China. Environmental Science and Technology, 42: 26742680.

Loska, K., Weichula, D., Barska, B., Cebula, E., Chojnecka, A. 2003. Assessment of arsenic enrichment of cultivated soil in southern Poland. Polish Journal of Environmental Studies, 12: 187-192.

Lu,X., Wang, L, Lei, K., Haung, J., Zhai, Y., 2009. Contamination assessment of copper, lead, zinc, manganese and nickel in street dust of Boaji, NW China. Journal of Hazardous Materials, 161: 186-192. 
Lu, X., Wang, L., Li, L.Y., Lei, K., Huang, L., Kang, D. 2010. Multivariate statistical analysis of heavy metals in street dust of Baoji, NW China. Journal of Hazardous Materials, 173: 744-749.

Marias, A.D., Blackhurst, D.M. 2009. Do heavy metals counter the potential health benefits of wine? Journal of Endocrinology, Metabolism and Diabetes of South Africa, JEMOSA, 14: 77-79.

Mashi, S.A., Yaro, S.A., Iyong, P.N. 2005. A survey of trends related to the contamination of street dust by heavy metal in Gwagwalada, Nigeria. Management of Environmental Quality, 16: 71- 76.

Massadeh, A.N., Snook, R.D. 2000. Determination of $\mathrm{Pb}$ and $\mathrm{Cd}$ in road dusts over the period in which $\mathrm{Pb}$ was removed from petrol in use. Journal of Environmental Monitoring, 4: 567-572.

Meglen, R.R. 1992. Examining large databases: a chemometric approach using principal component analysis. Marine Chemistry, 39: 217-237.

Meza-Figueroa, D., De La O-Villanueva, M., De La parra, M.L. 2007. Heavy metal distribution in dust from elementary schools in Hermosillo, Sonora, Mexico. Atmospheric Environment, 41: 276-288.

Muller, G. 1969. Index of geoaccumulation in sediments of the Rhine river. Geological Journal, 2: 108-118.

Papanikolaou, N.C, Hatzidaki, E.G, Belivanis, S., Tzanakakis, G.N., Tsatsakis, A.M. 2005. Lead toxicity update. a brief review. Medical Science Monitoring, 11: RA329-RA336.

Radojevic, M., Bashkin, V.N. 1999. Practical Environmental Analysis, 466 pp. Royal Society of Chemistry (RCS Publishing), Cambridge, UK.

Sheppard, D.S., Claridge, G.G.C., Campbell, I.B. 2000. Metal contamination of soils at Scoott Base, Antarctica. Applied Geochemistry, 15: 513-530.

Shinggu, D.Y., Ogugbuaja, V.O., Bariminas, J.T., Toma, I. 2007. Analysis of street dust for heavy metal pollutants in Mubi, Adamawa State, Nigeria. International Journal of Physical Sciences, 2: 290293.

Singh, K.P., Malik, A., Singh, S., Singh, V.K., Murphy, R.C. 2005. Estimation of source of heavy metal contamination in sediment of Gomti River (India) using principal component analysis. Water, Soil and Air Pollution, 166: 221-241.

Surthland, R.A., Tolosa, C.A., Tack, F.M.G., Verloo, M.G. 2000. Characterization of selected element concentration and enrichment ratios in background and anthropogenically impacted roadside areas. Archives of Environmental Contamination Toxicology, 38: 428-438.

Tamrakan, C.S., Shakya, P.R. 2011. Assessment of heavy metals in street dust in Kathmandu Metropolitan city and their possible impcts on the environment. Pakistan Journal of Analytical and Environmental Chemistry, 12: 34-41

Thomilson, D.C, Wilson, D.J, Harris, C.R, Jeffery D.W. 1980. Problem in heavy metals in estuaries and the formation of pollution index. Helgoländer Wissenschaftliche Meeresuntersuchungen, 33: 566575.

USEPA, 1989. United State Environment Protection Agency, Guidance Manual Assessing Human Health Risks from Chemically Contaminated, Fish and Shellfish, US Environmental Protection Agency, Washington, DC., USA.

Yap, C.K., Chew, W.Y., Tan, S.G. 2012. Heavy metal concentrations in ceiling fan and roadside car park dust collected from residential colleges in Universiti Putra Malaysia, Serdang, Selangor. Pertanika Journal of Tropical Agricultural Science, 35: 75-83.

Yetimoglu, E.K., Eracan, O., Tosyali, K. 2007. Heavy metal contamination in street dusts of Istanbul (Pendik to levent) E-5 Highway. Annali di Chimica, 97: 227-235.

Yomgming, H., Peixuan, D., Junji, C., Posmentior, E.S. 2006. Multivariate analysis of heavy metals contamination in urban dusts of Xi'an, Central China. The Science of the Total Environment, 355: 176-186.

Zhang, J., Liu, C. L. 2002. Riverine composition and estuarine geochemistry of the particulate metal in China-weathering features, anthropogenic impact and chemical fluxes. Estuarine and Coastal Shelf, Science, 54: 1051-1070. 